Bundesgesundheitsbl 2018 $61: 1453-1461$ https://doi.org/10.1007/s00103-018-2826-8 Online publiziert: 4. Oktober 2018 (C) Der/die Autor(en) 2018

CrossMark

Titus Josef Brinker, $\cdot$. Fabian Buslaff ${ }^{3}$. Caelán Haney ${ }^{4}$ Benedikt Gaim ${ }^{5}$. Ailís Ceara Haney ${ }^{4}$ - Selina Marisa Schmidt ${ }^{6}$. Marc Phillipp Silchmüller ${ }^{7}$. Lava Taha ${ }^{3} \cdot$ Lena Jakob $^{8} \cdot$ Hannah Maria Baumert ${ }^{8} \cdot$ Marvin Hallmann ${ }^{7}$. Marlene Heckl ${ }^{9}$. Jonas Alfitian ${ }^{10}$. Christian Martin Brieske ${ }^{11}$. Evgenia Petrova Divizieva ${ }^{12}$. Jilada Wilhelm ${ }^{12}$. Gabriel Hillebrand ${ }^{4}$. Dominik Penka ${ }^{13}$. Sanjeevan Raveendranathan ${ }^{14} \cdot$ Netzwerk Aufklärung gegen Tabak $^{4}$ · Janina Leonie Suhre ${ }^{15}$

'Abteilung für Translationale Onkologie, Nationales Centrum für Tumorerkrankungen (NCT), Deutsches Krebsforschungszentrum (DKFZ), Heidelberg, Deutschland; ${ }^{2}$ Klinik für Dermatologie,

Universitätsklinikum Heidelberg, Heidelberg, Deutschland; ${ }^{3}$ Universitätsklinikum Erlangen, Universität Erlangen, Erlangen, Deutschland; ${ }^{4}$ Universitätsklinikum Heidelberg, Universität Heidelberg, Heidelberg, Deutschland; ${ }^{5}$ Universitätsklinikum Regensburg, Universität Regensburg, Regensburg, Deutschland; ${ }^{6}$ Universitätsklinikum Tübingen, Universität Tübingen, Tübingen, Deutschland; ${ }^{7}$ Medizinische Hochschule Hannover, Universität Hannover, Hannover, Deutschland; ${ }^{8}$ Universitätsklinikum Freiburg, Universität Freiburg, Freiburg, Deutschland; ' ${ }^{9}$ nniversitätsklinikum München, Universität München, München, Deutschland; ${ }^{10}$ Universitätsklinikum Köln, Universität Köln, Köln, Deutschland;

${ }^{11}$ Universitätsklinikum Essen, Universität Essen, Essen, Deutschland; ${ }^{12}$ Universitätsklinikum Düsseldorf, Universität Düsseldorf, Düsseldorf, Deutschland; ${ }^{13}$ Universitätsklinikum Gießen, Universität Gießen, Gießen, Deutschland; ${ }^{14}$ Universitätsklinikum Münster, Universität Münster, Münster, Deutschland;

${ }^{15}$ Universitätsklinikum Bonn, Universität Bonn, Bonn, Deutschland

\title{
Das weltweite
} Medizinernetzwerk Aufklärung
gegen Tabak - Ehrenamtliche
Prävention made in Germany

im Bereich Tabakpräventionspolitik laut Weltgesundheitsorganisation (WHO) vor Österreich den vorletzten Platz in Europa [7]. So ist Deutschland z.B. das letzte Land in der Europäischen Union (EU), in dem Tabakaußenwerbung noch erlaubt ist $[8,9]$.

Vor diesem Hintergrund entstand 2012 das multinationale Netzwerk „Aufklärung gegen Tabak“ (AGT), auf Englisch: Education Against Tobacco (EAT). Im Folgenden werden die Initiative und ihr Konzept sowie die verschiedenen Aktivitäten des Netzwerks im Einzelnen vorgestellt, gefolgt von den Ergebnissen der durch AGT durchgeführten wissenschaftlichen Begleitstudien.

\section{Das Netzwerk „Aufklärung gegen Tabak"}

Das multinationale Netzwerk „Aufklärung gegen Tabak" ist eine von dem heutigen Arzt Dr. Titus J. Brinker im Jahr 2012 während des vorklinischen Teils seines Medizinstudiums gegründete Initiative, bei der sich inzwischen an circa $80 \mathrm{Me}$ dizinfakultäten in 14 Ländern über 3500 Medizinstudierende ehrenamtlich für die Tabakprävention engagieren (Stand: Mai 2018). AGT führt wissenschaftlich fundierte Präventionsseminare mit neuartigen Methoden durch. So finden zum Beispiel Facemorphing-Apps für das Smartphone Anwendung, die über 45.000 Schüler/innen pro Jahr erreichen. Zudem werden angehende Ärztinnen und Ärzte für die Problematik des Rauchens sensibilisiert. Zur Verbesserung der medizinischen Lehre wurden durch AGT wissenschaftlich fundierte Wahlfächer an 13 Medizinfakultäten in Deutschland initiiert. Diese sollen angehende Ärztinnen und Ärzte in der professionellen Tabakentwöhnung schulen, um ihren zahlreichen rauchenden Patientinnen und Patienten in Zukunft die bestmögliche Unterstützung beim 


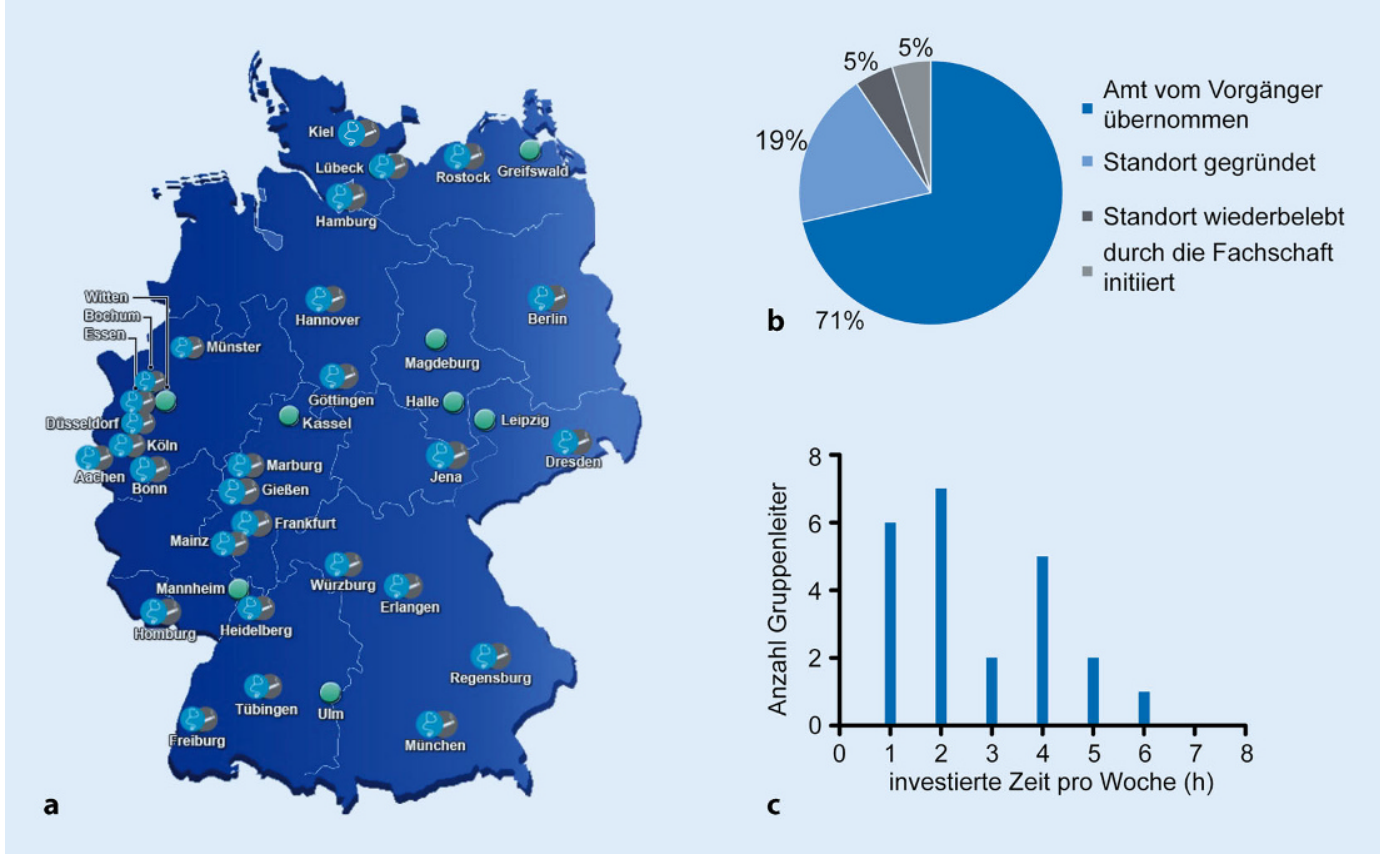

Abb. 1 < Lokalgruppen von „Aufklärung gegen Tabak" (AGT) an deutschen Medizinfakultäten. a AGTStandorte in Deutschland. Medizinfakultäten mit AGT-Lokalgruppen: AGT-Logo; ohne AGT-Lokalgruppe: grüner Punkt. b Lokalgruppenleitung. Ergebnisse der Gruppenleiterbefragung 2018 zu der Frage „Wie ist es dazu gekommen, dass du den Standort leitest?" $(n=23)$. c Zeitaufwand pro Woche. Ergebnisse der Gruppenleiterbefragung 2018 zu der Frage „Wie viele Stunden investierst du pro Woche in AGT?" $(n=23)$

Rauchstopp gewährleisten zu können. Bereits über 500.000 Raucherinnen und Raucher konnten kostenlos durch die selbstentwickelten Apps „Smokerface“ und „Smokerstop“ beim Aufhören unterstützt werden. Die Wirksamkeit der von AGT durchgeführten Maßnahmen wird durch Evaluationsstudien mit aktuell über 20.000 Sekundarschüler/innen stetig geprüft und verbessert. Sensibilisierte Medizinstudierende können nach ihrer Approbation im „Ärzteverband Tabakprävention" mit Politikern des Deutschen Bundestages in den Dialog treten [10-13].

\section{Handlungsebenen des Netzwerks zur Tabakprävention}

\section{Schulische Tabakprävention}

Die meisten Raucherinnen und Raucher beginnen ihren Tabakkonsum im frühen Jugendalter [14]. Laut einem Bericht der WHO aus dem Jahre 2016 rauchen in Deutschland $13 \%$ der Jungen und $15 \%$ der Mädchen bereits im Alter von 15 Jahren mindestens einmal in der Woche $\mathrm{Zi}$ garetten [15]. Bis zum jungen Erwachsenenalter steigt die Rauchprävalenz auf ungefähr ein Drittel [16]. Etwa die Hälfte der rauchenden Jugendlichen erfüllt bereits Kriterien der Nikotinabhängigkeit [17].
Vor diesem Hintergrund besucht AGT Schüler/innen der Jahrgangsstufen 6-8 aller Schultypen, um sie interaktiv und auf Augenhöhe über die Folgen des Rauchens aufzuklären. Sie sollen durch die Aufklärung eine bewusste eigenverantwortliche Entscheidung gegenüber dem Rauchen treffen. Die Einbindung von ehrenamtlichen Medizinstudierenden in das Programm bietet verschiedene Vorteile. Die Studierenden werden von den Jugendlichen durch den geringeren Altersabstand potenziell als authentischer und zugänglicher wahrgenommen als Lehrer oder Ärzte. Von Ärzten durchgeführte Präventionsprogramme zeigten bereits positive kurz- und langfristige Effekte [18, 19], allerdings sind diese Programme nicht vergleichbar breitenwirksam und kostengünstig wie AGT [18-20].

Das Präventionsprogramm der Initiative basiert auf einem sogenannten kombinierten Sozialeinfluss- und Sozialkompetenz-Ansatz („combined social influence and social competence approach“), der in der Literatur als erfolgversprechendster Weg in der schulbasierten Tabakprävention beschrieben wird [21]. Auf altersgerechte Inhalte und Interaktivität wird besonderer Wert gelegt, da es wissenschaftliche Hinweise auf eine erhöhte Motivation $\mathrm{zu}$ einem Aufhörversuch gibt, wenn die Person die gesundheitlichen Folgen selbst erarbeiten muss, anstatt sie vorgetragen $\mathrm{zu}$ bekommen [22].

ProSchuljahr betreut AGT im deutschsprachigen Raum mit etwa 1500 Medizinstudierenden aktuell ca. 23.800 Schüler/ innen (durchschnittlich 700 Schüler/ innen pro Universität, 28 Universitäten in Deutschland (- Abb. 1a), 4 in Österreich, 2 in der Schweiz). Die Lokalgruppen organisieren sich selbstständig, die Gruppenleitung übergibt ihre Funktion bei Abschluss des Studiums an jüngere Studierende. So bestehen die meisten Lokalgruppen bereits erfolgreich in mindestens der zweiten Generation (-Abb. 1b). Die Gruppenleiter investieren ihre Zeit ehrenamtlich in die Koordination der Lokalgruppen (- Abb. 1c und 2).

\section{Smokerface-App und Mirroring- Ansatz}

Das selbst wahrgenommene Aussehen ist der stärkste Prädiktor für Selbstbewusstsein im Jugendalter [23]. Burford et al. konnten in einer randomisierten kontrollierten Studie nachweisen, dass ein mittels Desktop-Programm verändertes Selbstporträt (=Selfie), welches das eigene Gesicht als Raucher/in vs. Nichtraucher/in mehrere Jahre in der Zukunft zeigt, signifikante Auswirkun- 
Bundesgesundheitsbl 2018 $61: 1453-1461$ https://doi.org/10.1007/s00103-018-2826-8

(c) Der/die Autor(en) 2018

T. J. Brinker · F. Buslaff · C. Haney · B. Gaim · A. C. Haney · S. M. Schmidt · M. P. Silchmüller · L. Taha · L. Jakob · H. M. Baumert · M. Hallmann · M. Heckl · J. Alfitian · C. M. Brieske · E. P. Divizieva · J. Wilhelm · G. Hillebrand · D. Penka · S. Raveendranathan · Netzwerk Aufklärung gegen Tabak.J. L. Suhre

\section{Das weltweite Medizinernetzwerk Aufklärung gegen Tabak - Ehrenamtliche Prävention made in Germany}

\section{Zusammenfassung}

Das Rauchen ist in Deutschland die größte vermeidbare Ursache für frühzeitigen Tod. Das Netzwerk „Aufklärung gegen Tabak" (AGT) ist eine im Jahr 2012 in Deutschland gegründete Initiative, bei der sich inzwischen in 14 Ländern an circa 80 Medizinfakultäten über 3500 Medizinstudierende, Ärztinnen und Ärzte ehrenamtlich engagieren. In diesem Beitrag stellt die Initiative AGT ihr Konzept, sowie Aktivitäten, Ziele und begleitende Studien vor.

Auf Schulebene adressiert das Programm 10- bis 15-jährige Sekundarschüler und -schülerinnen. Ergänzend zu einem multimodalen Ansatz werden bei den Schulbesuchen moderne Medien wie Facemorphing-Apps eingesetzt, die nicht nur von Schülern (45.000 pro Jahr in 14 Ländern), sondern insgesamt bereits von über 500.000 Menschen genutzt werden. Die Wirksamkeit der schulbasierten Intervention wird in randomisierten Langzeitstudien mit aktuell 20.000 Jugendlichen in Deutschland untersucht. Eine erste Langzeitstudie zeigte Hinweise auf einen protektiven Effekt bezüglich Rauchbeginn, besonders bei Schülerinnen, niedrigem Bildungsniveau und vorhandenem Migrationshintergrund.

An 13 (von 28 teilnehmenden) deutschen Medizinfakultäten bildet das Programm jährlich mehrere hundert angehende Ärztinnen und Ärzte in wissenschaftlich konzipierten Wahlfächern für die fundierte Entwöhnung von Patienten aus und sensibilisiert sie für die Tabakepidemie. Approbierte Mitglieder treten als „Ärzteverband Tabakprävention" mit lokalen Bundestagsabgeordneten in den Dialog.

AGT motiviert die angehende Medizinergeneration, initial über Prävention im Schulsetting, sich der Herausforderung der nationalen Tabakkontrolle auch auf hochschulpolitischer und bundespolitischer Ebene zu stellen.

\section{Schlüsselwörter}

Medizinstudierende · Jugendliche · Schulbasierte Tabakprävention · Tabakentwöhnung · Tabakkontrolle

\section{The global medical network Education Against Tobacco—voluntary tobacco prevention made in Germany}

\section{Abstract}

Smoking is the leading preventable cause of premature death in Germany. The network "Education Against Tobacco" (EAT) is an initiative that was founded in Germany in 2012, in which more than 3500 medical students and physicians engage in volunteer work in about 80 medical faculties in 14 countries. In this article, the concept, activities, objectives and associated research studies oft he EAT initiative are introduced. On the school level, the initiative addresses 10- to 15-year-old secondary school students. In addition to a multimodal approach, school visits use modern media such as facemorphing apps, which are not only used by students ( 45,000 per year in 14 countries), but by a total of over 500,000 other people as well. The effectiveness of the school-based intervention is currently being investigated in randomised long-term studies with 20,000 adolescents in Germany. A first long-term study demonstrated evidence of a protective effect regarding the onset of smoking, especially among female students, students having a low level of education and students with a migratory background.

The programme educates several hundred prospective physicians at 13 (of 28 participating) German medical faculties each year in science-based elective courses for the wellestablished smoking cessation counselling of patients and sensitises them to the tobacco epidemic. The approved members engage in dialogue with local members of the German house of representatives as "Ärzteverband Tabakprävention".

EAT motivates the prospective generation of physicians, initially through prevention in school settings, to face the challenge of national tobacco control at the university and federal level.

\section{Keywords}

Medical students · Adolescents · School-based tobacco prevention - Smoking cessation . Facemorphing apps gen auf die 6-Monats-Prävalenz bei 18bis 30-jährigen jungen Erwachsenen hat (Abstinenz in der Interventionsgruppe nach sechs Monaten $=27,5 \%$ vs. Kontrollgruppe $=6,3 \%$ ). Auch die Aufhörmotivation von 14- bis 18-jährigen Frauen konnte durch das Computerprogramm gesteigert werden [24]. Allerdings sind diese Programme oft teuer und nicht in der Breite verfügbar.

Das starke Interesse der Jugendlichen für ihr Aussehen führte zu der
Idee, die Smokerface-App zu entwickeln [23]. Diese verändert grafisch ein Selfie des Nutzers und prognostiziert die Veränderungen im Gesicht, die durch das Rauchen einer Zigarettenpackung pro Tag zu verschiedenen zukünftigen Zeitpunkten (nach 1, 3, 6, 9, 12 oder 15 Jahren) im Vergleich zum Nichtrauchen entstehen würden (• Abb. 3). Die App-Algorithmen beachten das aktuelle Alter des Nutzers und verändern das Gesicht unter Berücksichtigung publi- zierter Auswirkungen des Rauchens auf das Aussehen, wie zum Beispiel ein erhöhtes Aknerisiko oder blassere Haut durch verminderte Kapillarperfusion (bereits nach einem Packungsjahr), sowie Bindegewebsschäden und Falten [25, 26].

Zunächst war die Smokerface-App nur als Computerversion in $2 \mathrm{D}$ verfügbar. Um sie noch breiter nutzbar $\mathrm{zu}$ machen, wurde die App auch für Smartphones herausgebracht, von denen fast 

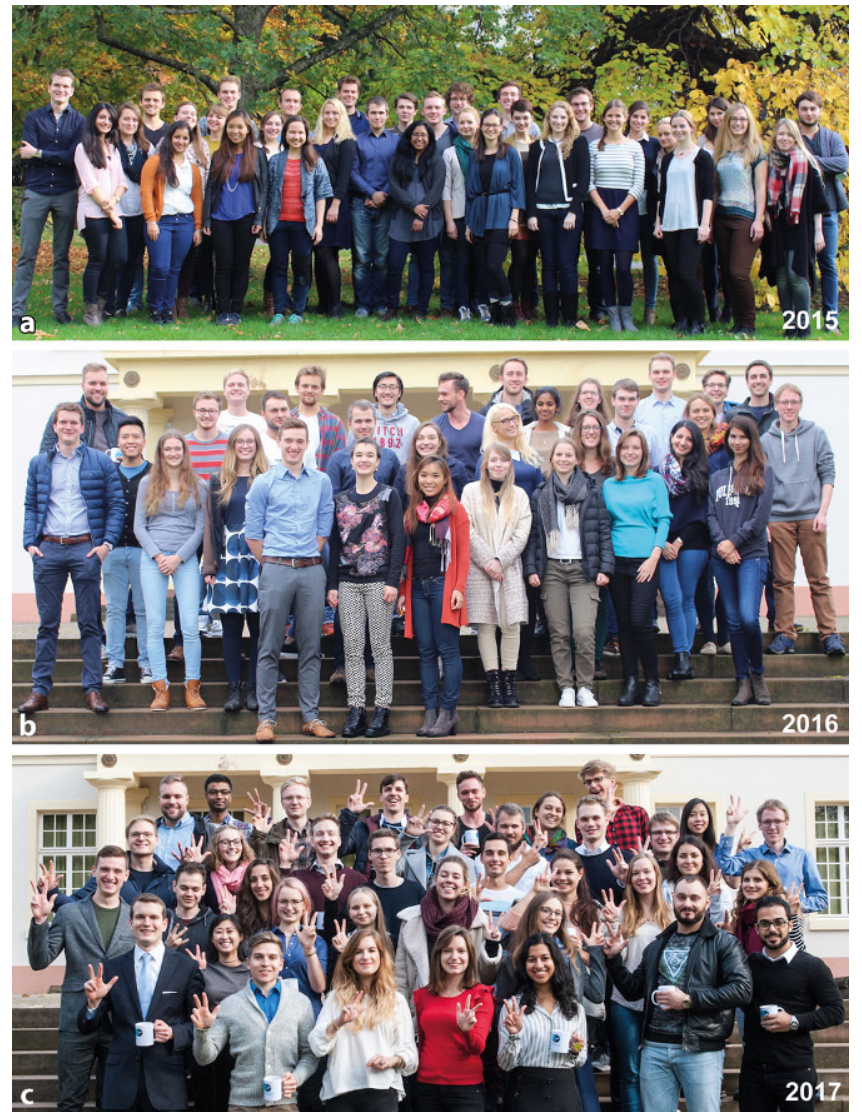

Abb. $2 \triangleleft$ Gruppenleiter von „Aufklärung gegen Tabak" auf dem Bundestreffen in Heidelberg. a Bundestreffen 2015. b Bundestreffen 2016. c Bundestreffen 2017

jeder Jugendliche eines besitzt [27]. Des Weiteren wurde die App kontinuierlich verbessert und ist nun mit 3D-Animationen verfügbar. Der Nutzer kann mit seinem eigenen Smokerface durch Berührung interagieren. Über eine Erklärgrafik erläutert die App weiterhin die positiven Aspekte des Nichtrauchens und die negativen Auswirkungen des Rauchens anhand der Gegenüberstellung eines Nichtrauchergesichtes und eines frühzeitig gealterten Rauchergesichtes [12]. Die im Internet frei und kostenlos zugängliche, in die sechs meistgesprochenen Sprachen übersetzte App wird derzeit ca. 100-mal pro Tag heruntergeladen.

Um die Nutzung der App im Schulsetting stärker zu fokussieren, wurde der Mirroring-Ansatz entwickelt: Anstatt der bisher verwendeten Computerversion wird nun die neuere SmartphoneVersion genutzt und das dreidimensionale Selfie mithilfe einer MirroringSoftware vom Smartphone des Schülers oder von zur Verfügung gestellten Tablets über den Beamer auf eine Leinwand übertragen. Somit haben alle Mitschüler/innen (und auch die Lehrer/innen) die Möglichkeit, auf das Bild zu reagieren. Diese Reaktionen beeinflussen die subjektive Norm, welche einen Einfluss auf jugendliches Rauchverhalten hat [28]. Die Funktionsweise der App wird anhand eines freiwilligen Jugendlichen erklärt, und die Schüler/innen werden aufgefordert, die ihnen aufgefallenen Veränderungen zu beschreiben. Die Medizinstudierenden erklären ihnen im Anschluss, weshalb das Rauchen diese verursacht.

\section{Die Smokerface-Posterkampagne:} eine Alternative zu angeleiteten Präventionsprogrammen?

Die meisten effektiven schulischen Tabakpräventionsprogramme benötigen externe Mentoren und sind daher nicht in der Breite verfügbar. Des Weiteren sind solche Interventionen oft mit hohen Kosten und organisatorischem Aufwand verbunden, was eine großflächige Implementierung in Schulen behindert
[21]. Auch wenn das AGT-Programm durch das ehrenamtliche Engagement von Medizinstudierenden günstiger und breitenwirksamer ist als von Ärztinnen und Ärzten durchgeführte Präventionsprogramme, sind die Kapazitäten dennoch durch die zeitliche Verfügbarkeit der Medizinstudierenden limitiert. Daher wurde eine weitere Implementationsmethode entwickelt, die davon unabhängig ist: Eine die FacemorphingApp bewerbende Posterkampagne ist eine günstige (weniger als $50 €$ pro Schule), breitenverfügbare, einfach $\mathrm{zu}$ verwendende Alternative oder Ergänzung zu den bisherigen Präventionsprogrammen.

Die Poster illustrieren die Auswirkungen des Rauchens auf das Gesicht anhand einer Gegenüberstellung des zukünftigen Nichtrauchergesichts und des vorzeitig gealterten Rauchergesichts (• Abb. 3). Des Weiteren enthält das Poster eine Aufforderung an die Jugendlichen, sich die kostenlose App herunterzuladen und herauszufinden, wie ihr persönliches Smokerface aussehen würde.

\section{Wahlfach Tabakprävention}

Die bisher vorgestellten Aktivitäten von AGT verfolgen einen überwiegend primärpräventiven Ansatz, denn der größte gesundheitliche Nutzen für eine Person wird ohne Frage dann erzielt, wenn die Nikotinsucht primär nicht einsetzt und nicht therapiert werden muss [4]. Dennoch ist der Rauchstopp sekundär- und sogar noch tertiärpräventiv oft die effektivste Maßnahme, um tabakbedingte Folgeerkrankungen $\mathrm{zu}$ verhindern oder den Verlauf bereits eingetretener Erkrankungen zu beeinflussen [29].

Trotz der enormen Wichtigkeit der Tabakentwöhnung zeigen Studien, dass die Tabakabhängigkeit im Vergleich zu anderen chronischen Erkrankungen wie Diabetes oder Hypertension nicht adäquat behandelt wird [30-32]. Als mögliche Ursachen werden fehlende Motivation, nicht trainierte Fertigkeiten und Unkenntnis von geeigneten Beratungsstrategien und Behandlungsmethoden genannt [30-33]: Im Rahmen mehrerer an deutschen Medizinfakultäten durchgeführter Studien konnten sich weniger als $5 \%$ der Studierenden 


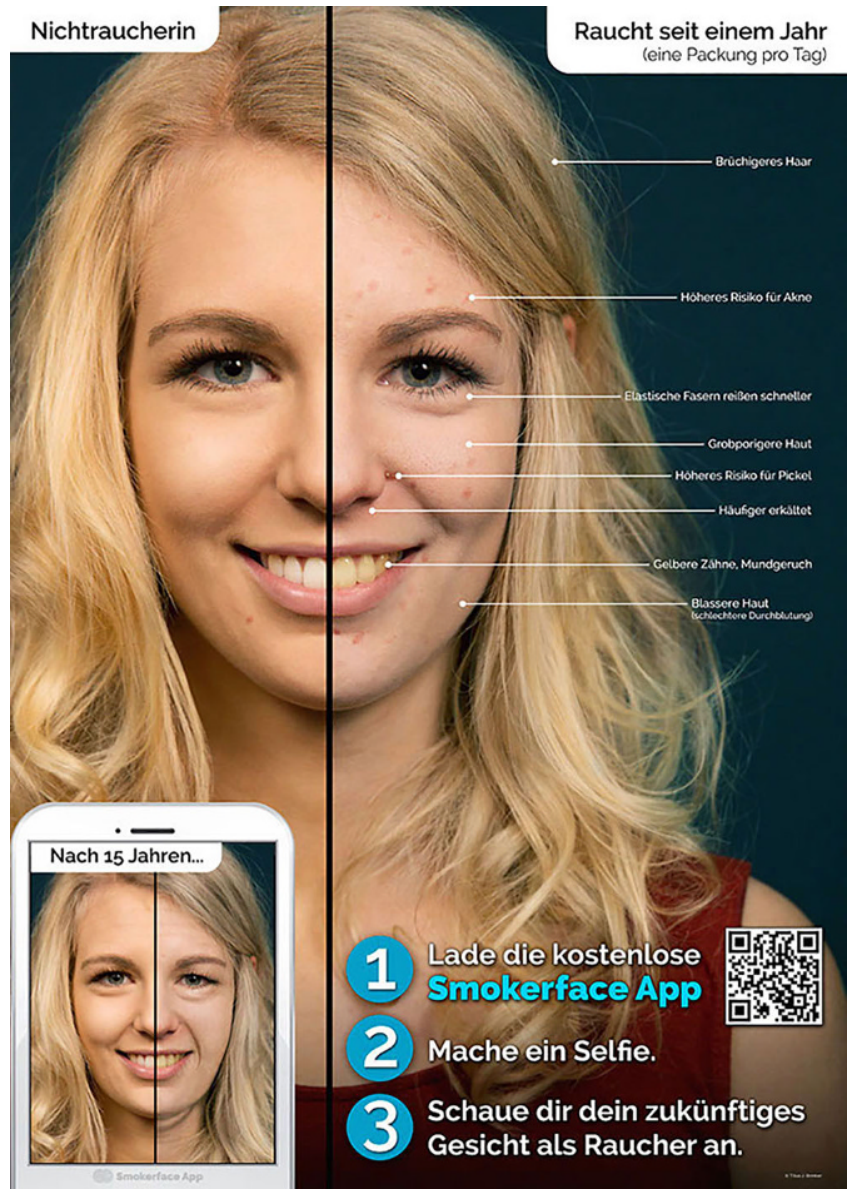

Abb. $3<$ Smokerface-App-bewerbendes Poster, welches nach erfolgter Intervention aufgehängt wird. Gezeigt werden die Effekte der App an einem Beispielgesicht. Linke Gesichtshälfte: jeweils Alterung als Nichtraucher/in; rechte Gesichtshälfte jeweils Alterung bei Konsum von einem Päckchen Zigaretten pro Tag

im letzten Studienjahr überhaupt daran erinnern, jemals praktisch zur Beratung von Raucher/innen angeleitet worden zu sein [34], und vielen Studierenden war nicht bekannt, welche Behandlungsmethoden effektiv sind und welche nicht [35]. Über die Hälfte der Studierenden wünschte sich eine Intensivierung der Lehre zur Tabakabhängigkeit [36].

Die Ausbildung Medizinstudierender hinsichtlich der Beratung und Behandlung rauchender Patientinnen und $\mathrm{Pa}$ tienten ist somit verbesserungswürdig. Um diese Missstände zu beheben, wurde durch AGT an 13 Standorten ein wissenschaftlich-konzipiertes Wahlfach eingeführt. Die Studierenden erlernen unter anderem Elemente des $5 \mathrm{~A}$-Schemas zur Raucherberatung (Ask, Advise, Assess, Assisst, Arrange) und die Effektivität ärztlicher Maßnahmen und Unterstützungsmethoden. Diese werden auch an Schauspielpatient/innen praktisch erprobt. Des Weiteren nehmen die Studierenden im Rahmen des Wahlfachs an einem Schulbesuch teil. Am Ende des enten bezüglich ihrer Tabakabhängigkeit besser betreuen können.

\section{Ärzteverband Tabakprävention}

Ein starker Fokus der Maßnahmen von AGT liegt auf individueller Verhaltensprävention. Diese ist ungemein wichtig; einen größeren Erfolg verspricht jedoch eine Kombination mit einer konsequenten Verhältnisprävention, um die äußeren Umstände, die zu der Entstehung von Tabakabhängigkeit und tabakbedingten Folgeerkrankungen beitragen, zu verändern.

Das Ziel von AGT ist es daher, die Verbreitung der tabakbedingten chronischen Erkrankungen durch den Anstoß legislativer Maßnahmen zu reduzieren. Mittels ehrenamtlicher Einflussnahme sollen Ärzte und Ärztinnen mit lokalen Mitgliedern des Bundestags in den Dialog treten und die politischen Entscheidungsträger überzeugen, ein gesetzlich verankertes Spitzenniveau in der Tabakprävention anzustreben. Von diesem Spitzenniveau ist Deutschland weit entfernt, zurzeit ist es eines der rückständigsten Länder in Europa. Deutschland belegt den vorletzten Platz auf der Tabakkontrollskala, welche die nationale Implementation von politischen, wissenschaftlich fundierten Maßnahmen zur Senkung der Rauchprävalenz misst. [7].

Durch den Kontakt mit der Politik soll eine positive Gegenstimme zur Tabakindustrie die Umsetzung der bereits im Jahr 2003 von Deutschland unterzeichneten, im Rahmenübereinkommen der WHO zur Tabakkontrolle geforderten Maßnahmen fördern. Die Herangehensweise hierbei orientiert sich an international erfolgreichen Ärzteverbänden. Die Forderungen beinhalten ein vollständiges Tabakwerbeverbot, die Erstattung der Kosten von Tabakentwöhnungsmaßnahmen durch die Krankenkassen, eine Erhöhung der Tabaksteuer, Verbesserung des Nichtraucherschutzes und bessere Aufklärung der Bürger über die Folgen des Tabakkonsums. Medizinstudierende, die durch AGT während ihres Studiums für die Thematik sensibilisiert wurden, können ihr Engagement im Ärzteverband fortsetzen. Auch approbierte 


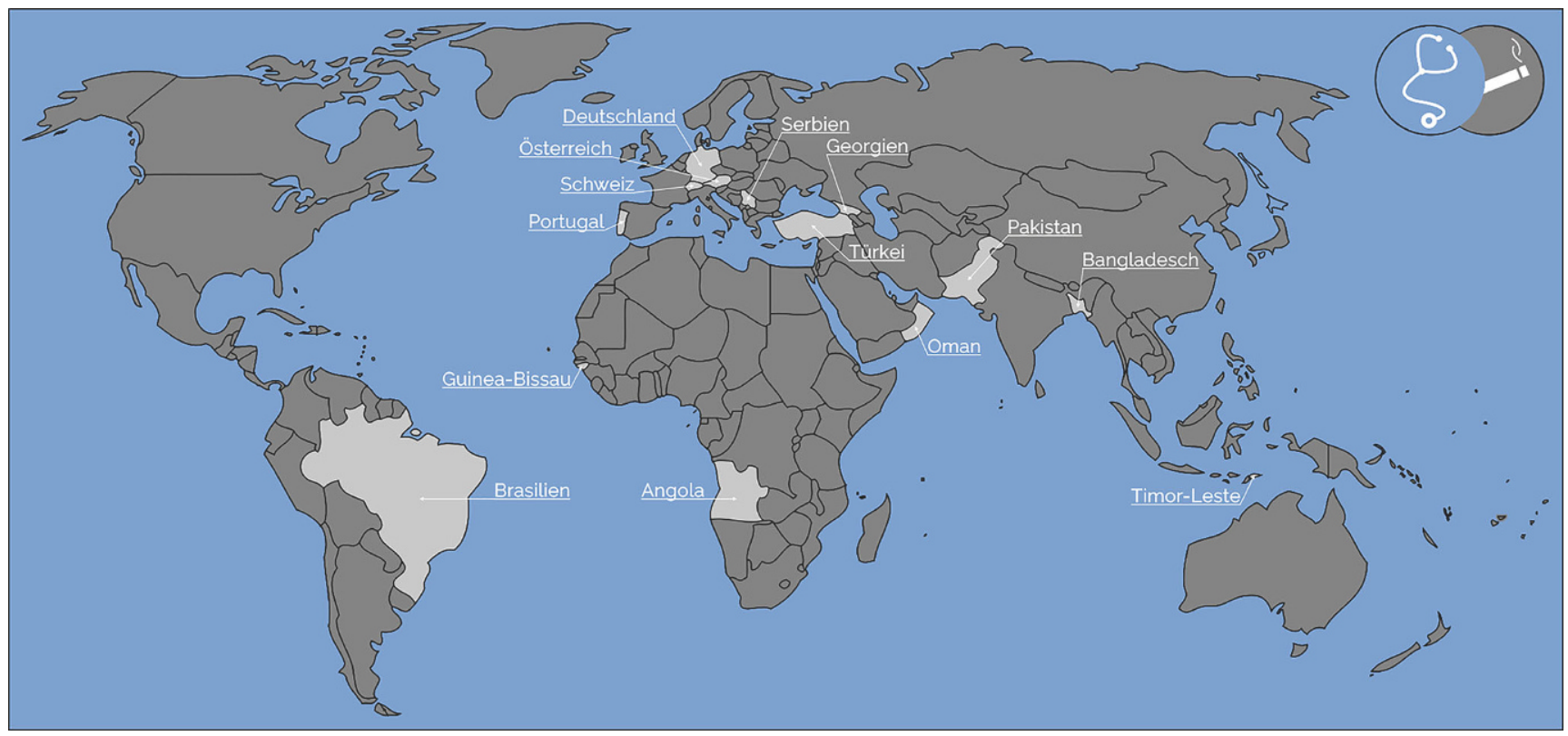

Abb. 4 \ Weltweite Verbreitung von „Aufklärung gegen Tabak“ (Stand: April 2018). In den hellgrau markierten Ländern befinden sich Medizinfakultäten, an denen das Programm implementiert wurde. Oben rechts: einheitliches Logo. Mit freundlicher Genehmigung von Selina Schmidt und Benedikt Gaim (Vorstandskoordination Europa)

Ärztinnen und Ärzte, die keine AGTAlumni sind, können mitwirken.

\section{Internationalisierung des} Programms ausgehend von der Schulebene

Die Problematik der Tabakabhängigkeit ist nicht aufDeutschland begrenzt. Daher wurde das zu Beginn nur in deutschsprachigen Ländern verfügbare Programm von AGT in verschiedene Sprachen übersetzt und findet nun an über 80 Medizinfakultäten in 14 Ländern Anwendung. Derzeit werden durch die Standorte auBerhalb des deutschsprachigen Raumes etwa 21.000 Schüler/innen erreicht. Die Länder, in denen Medizinfakultäten das Programm implementiert haben, werden in $-A b b .4$ gezeigt. Die internationalen Standorte organisieren sich selbstständig und arbeiten an einer Ausweitung des Programms innerhalb der eigenen Landesgrenzen sowie an einer Expansion in gleichsprachige Länder. Auch werden über die schulbasierte Aktivität hinaus eigene Ideen und Initiativen lanciert, wie beispielsweise ein Social-Media-Wettbewerb auf Facebook mit der SmokerfaceApp zum Weltnichtrauchertag 2017 in Pakistan oder die Ausschreibung eines Awards für Tabakkontrolle für die beste
Idee zur Senkung der Rauchprävalenz in Brasilien.

\section{Wissenschaftliche Begleitung - Ergebnisse der Evaluations- studien}

\section{Schulische Tabakprävention}

Um die Wirksamkeit des durch AGT entwickelten Präventionsprogramms zu untersuchen und zu verbessern, werden durch AGT die deutschlandweit größten Multicenterstudien zum Thema schulische Tabakprävention durchgeführt.

Eine erste prospektive, quasi-experimentelle Studie mit etwa 1500 Jugendlichen zur Wirksamkeit der Schulpräventionsprogramme auf die Reduktion der Rauchprävalenz bei 11- bis 15-jährigen Schüler/innen wurde bereits 2014 von AGT durchgeführt $[10,37]$. Die Intervention bestand aus dem ursprünglichen zweistündigen Präventionsprogramm von AGT. Ein Patient mit einer tabakbedingten Erkrankung konnte von den Jugendlichen bezüglich seiner Beweggründe und Erfahrungen mit dem Rauchen befragt werden. Es konnten signifikante Effekte auf den Rauchstopp (=sekundärpräventive Effekte) nachgewiesen werden. Die Intervention hatte nach einem halben Jahr einen stärkeren sekundärpräventiven Effekt auf Gymnasiasten als auf Gesamtschüler/innen. Ein primärpräventiver Effekt (Raucheinstieg) konnte nach sechs Monaten nicht festgestellt werden [10].

Aufgrund der Ergebnisse dieser Studie wurde das Curriculum für Schultypen mit niedrigerem Bildungsniveau (Gesamt-/Hauptschulen) unter Einbezug eines Schülerfeedbacks angepasst und nach neuen Ansätzen gesucht, die diese Jugendlichen besonders ansprechen könnten. Der Fokus wurde verstärkt auf die Vorteile des Nichtrauchens gelegt, anstatt das Rauchen abzuwerten, da Studien zeigen konnten, dass ärztliche Präventionsprogramme mit einem Schwerpunkt auf Abschreckung und Angstinduktion keinen Langzeiteffekt auf die Reduktion der Rauchprävalenz aufwiesen [20, 39-42]. Im Gegensatz dazu konnte ein ärztliches multimodales Präventionsprogramm in Berlin in einer quasi-experimentellen Studie signifikante Kurzzeiteffekte auf den Rauchbeginn aufzeigen. Dies könnte eine vielversprechende Alternative zu dem traditionell genutzten Abschreckungsansatz darstellen [19]. Die von AGT entwickelte Smokerface-App wurde in der ursprünglichen $2 \mathrm{D}$-Computerversion 
erstmals in das Curriculum eingebaut, und jeder Jugendliche erhielt sein eigenes überarbeitetes Selfie [10]. Die randomisierte Studie mit einem Jahr Followup prüfte die Effektivität des überarbeiteten Curriculums hinsichtlich der Prävention des Rauchens bei 11- bis 15jährigen Schüler/innen [43]. Zusammenfassend zeigte diese erste Langzeitstudie zu den AGT-Präventionsprogrammen mit Smokerface-Intervention Hinweise auf einen protektiven Effekt bezüglich Rauchbeginn, besonders bei weiblichem Geschlecht (Number Needed to Treat $(\mathrm{NNT})=24)$, bei niedrigem Bildungsniveau $(\mathrm{NNT}=30$, im Gegensatz zu NNT $=199$ bei Gymnasien) oder bei Migrationshintergrund $(\mathrm{NNT}=44)$. Dies lässt sich mit anderen Studien vereinbaren, die eine besondere Bedeutung der selbstwahrgenommenen Attraktivität als stärksten Einfluss auf das Selbstbewusstsein, vor allem für Mädchen oder Schüler/innen niedrigerer Bildungsstufen beimessen [23]. Sekundärpräventive Effekte wie eine Motivation zum Rauchstopp konnten in dieser Studie nicht nachgewiesen werden.

Der Mirroring-Ansatz, bei dem das Smokerface eines freiwilligen Jugendlichen vor die gesamte Peergroup projiziert wird, wurde an drei weiterführenden Schulen getestet. Direkt nach der Intervention wurden dieSchüler/innen mittels eines anonymen Fragebogens nach ihrer Meinung $\mathrm{zu}$ der Intervention gefragt. Die meisten Jugendlichen gaben an, die Intervention habe ihnen Spaß gemacht $(77 / 125,61,6 \%)$, sie zum Nichtrauchen motiviert (79/125, 63,2\%), ihnen bisher unbekannte Vorteile des Nichtrauchens vermittelt $(81 / 125,64,8 \%)$. Nur eine Minderheit verneinte es, teilweise oder völlig neue Vorteile des Nichtrauchens gelernt zu haben $(16 / 125,12,8 \%)$ oder zum Nichtrauchen motiviert worden zu sein $(18 / 125,14,4 \%) .90 \%$ der Jugendlichen hielten die App für eine geeignete Maßnahme, um ihre Mitschüler/innen vom Nichtrauchen zu überzeugen [11].

Im Jahr 2016 wurde das AGT-Curriculum nach den Erkenntnissen aus den eigenen Studien und anderen aktuellen Forschungsergebnissen erneut optimiert. Elemente zur Verstärkung von Gruppeneffekten (wie der Mirroring-Ansatz) und zur Repetition des Gelernten (wie zum Beispiel App-bewerbende Poster [• Abb. 3]) wurden integriert. Langzeitfolgen für die Gesundheit wurden aufgrund ihrer Ineffektivität komplett aus dem Curriculum genommen [44]. Es handelt sich dabei um die aktuellste Version, wie sie derzeit in den Klassenräumen durchgeführt wird (Stand: Mai 2018). Ihre Wirksamkeit wird in einer momentan noch andauernden kontrolliert randomisierten Studie mit ca. 10.000 Siebtklässler/innen in 140 Sekundarschulen untersucht. Als primärer Endpunkt ist der Unterschied (Kontroll- vs. Interventionsschulen) in der Veränderung des Anteils an Raucher/ innen und Nichtraucher/innen nach 24 Monaten im Vergleich zur Baseline definiert („difference of differences approach“). Der sekundäre Endpunkt ist die Veränderung der Einstellungen der Jugendlichen zum Rauchen (basierend auf der Theorie des geplanten Verhaltens) in den beiden Studienarmen nach 24 Monaten im Vergleich zur Baseline.

\section{Smokerface-Posterkampagne}

Die Wirksamkeit der Posterkampagne wird derzeit in einer randomisierten kontrollierten Studie evaluiert [45]. 9851 Schüler/innen von 126 weiterführenden Schulen nehmen an der prospektiv experimentellen Studie teil. Die Datenerhebung erfolgt mittels Fragebogen zu Beginn, sowie 6, 12 und 24 Monate nach Intervention. Die Abschlussbefragung wird durch zufällig ausgewählte Kohlenmonoxid-Atemtests zur biochemischen Validierung der Ergebnisse ergänzt. Die Interventionsschulen erhalten die Smokerface-Posterkampagne, Kontrollschulen mit vergleichbaren Baseline-Daten erhalten keine Intervention. In der Baseline-Befragung war die Prävalenz für Zigarettenrauchen $4,7 \%$, für E-Zigaretten $4,6 \%$ ( $1,6 \%$ nutzen beides). Der primäre Endpunkt ist die Differenz der Rauchprävalenz in der Nachbefragung nach 24 Monaten in der Kontrollgruppe im Vergleich zur Interventionsgruppe. Als sekundäre Endpunkte werden Veränderungen in den Einstellungen zum Rauchen, sowie die Zahl der Jugendlichen, die das Rauchen neu begonnen oder beendet haben, gemessen. Die Datenerhebung wird im August 2018 abgeschlossen und die Ergebnisse im Anschluss präsentiert und zur Publikation eingereicht.

\section{Internationale Verbreitung und Generalisierbarkeit des Programms}

$\mathrm{Da}$ in einer vergangenen Studie bereits die unterschiedliche Wirksamkeit eines Tabakpräventionsprogramms in verschiedenen europäischen Ländern gezeigt werden konnte, kann angenommen werden, dass auch das AGT-Curriculum vor verschiedenen soziokulturellen Hintergründen nicht generalisiert präventiv wirkt $[34,46]$. Daher werden parallel zu der Evaluation in Deutschland der Mirroring-Ansatz sowie das komplette Curriculum in randomisiert kontrollierten Langzeitstudien mit über 1500 Sekundarschüler/innen in Brasilien untersucht $[13,47]$.

\section{Ausblick}

Zu Beginn fokussierte sich das Engagement von AGT auf die schulische Tabakprävention in Deutschland. Anhand der derzeit noch ausstehenden Ergebnisse der laufenden Evaluationsstudien wird das Programm nach neuesten Erkenntnissen auch in Zukunft immer wieder verbessert. Auch eine Übertragbarkeit auf andere soziokulturelle Hintergründe ist Bestand aktueller Evaluationen. Die internationale Verbreitung und Implementierung des Programms weitet sich stetig aus. Auch auf Tabakkontrollebene nehmen die Aktivitäten national und international zu: Der Ärzteverband Tabakprävention versucht über einen politischen Dialog Verhältnisprävention zu betreiben (z. B. Erhöhung der Tabaksteuer, Tabakwerbeverbot) und gewinnt stetig an Mitgliedern. In Zukunft wird eine Ausweitung des Programms auf neue Zielgruppen wie zum Beispiel Krankenpflegeschüler/innen evaluiert, um auch verhaltenspräventive Effekte in einer neuen Zielgruppe mit deutlich höherer Rauchprävalenz weiter zu steigern. Vor diesem Hintergrund wurde eine Übertragbarkeit des Mirroring-Ansatzes mit 197 Pflegeschüler/innen im April 2018 in 
Köln und Bonn getestet (Raucherquote 27,3\%; [48]) Anhand dieser Ergebnisse soll ein neues, für diese Altersgruppe zugeschnittenes Curriculum entwickelt, national sowie international ausgerollt und evaluiert werden.

\section{Korrespondenzadresse}

\section{Dr. med. Titus Josef Brinker}

Abteilung für Translationale Onkologie, Nationales Centrum für Tumorerkrankungen (NCT), Deutsches Krebsforschungszentrum (DKFZ) Im Neuenheimer Feld 460, 69120 Heidelberg, Deutschland titus.brinker@nct-heidelberg.de

Collaborators. Ole Anhuef (Tübingen), Pascal Marc Eberz (München), Daniel Zeymer von Metnitz (Tübingen), Fabian Norbert Fries (Homburg), Franziska Sigrid Maser (Jena), Julia Holzapfel (Jena), Felix Johannes Hofmann (Gießen), Sonja Valeska Baumermann (Bochum), Thorben Royeck (Münster), Hanna Beißwenger (Göttingen), Gabriel Hillebrand (Heidelberg), Mohamed Barghout (Lübeck), Aycen Koc (Berlin), Fabian Schneeberg (Bern), Martina Zangger (Bern), Carla Bauermeister (Dresden), Breno Bernardes-Souza (Brasilien), Rayanna Mara O. S. Pereira (Brasilien), Sara Helena Ksiazek (Wien), Anna Nagel (Wien), Lilian Diem (Innsbruck), Prof. Dr. Anil Batra (Tübingen), Prof. Dr. Veronika Grau (Gießen), Prof. Dr. Wolfgang Kummer (Gießen), Prof. Dr. David A. Groneberg (Frankfurt), Prof. Dr. Werner Seeger (Gießen), PD Dr. Ute Mons (Heidelberg), Prof. Dr. Felix Herth (Heidelberg), Prof. Dr. Michael Kreuter (Heidelberg), Dr. Claudia M. Bauer (Heidelberg), Prof. Dr. Christof von Kalle (Heidelberg), Aayushi Srivastava (Heidelberg)

Danksagung. Besonders dankbar sind wir unseren Förderern: der Herzstiftung, dem Deutschen Zentrum für Lungenforschung, der Lungenstiftung und der Krebshilfe. Diese Institutionen ermöglichen uns unsere Forschungstätigkeit und unterstützen die Qualitätssicherung unserer Interventionen. Danken möchten wir auch allen Medizinstudierenden, die sich ehrenamtlich in dem Projekt engagieren.

Ganz besonders danken wir den unter Collaborators aufgeführten Personen, die dem Netzwerk Aufklärung gegen Tabak (AGT) in leitenden Funktionen angehör(t)en.

\section{Einhaltung ethischer Richtlinien}

Interessenkonflikt. T. J. Brinker, F. Buslaff, C. Haney, B. Gaim, A.C. Haney, S. M. Schmidt, M. P. Silchmüller, L. Taha, L. Jakob, H.M. Baumert, M. Hallmann, M. Heckl, J. Alfitian, C. M. Brieske, E. P. Divizieva, J. Wilhelm, G. Hillebrand, D. Penka, S. Raveendranathan und J. L. Suhre geben an, dass kein Interessenkonflikt besteht.

Den Empfehlungen zur Sicherung der Guten Epidemiologischen Praxis (GEP) folgend, wurden vor den Datenerhebungen Ethikvoten und Genehmigungen der verantwortlichen Institutionen eingeholt. Die
Schüler/innen und Eltern erklärten nach Information schriftlich ihr Einverständnis.

Open Access. Dieser Artikel wird unter der Creative Commons Namensnennung 4.0 International Lizenz (http://creativecommons.org/licenses/by/4.0/deed. de) veröffentlicht, welche die Nutzung, Vervielfältigung, Bearbeitung, Verbreitung und Wiedergabe in jeglichem Medium und Format erlaubt, sofern Sie den/die ursprünglichen Autor(en) und die Quelle ordnungsgemäßnennen, einen Linkzur Creative Commons Lizenz beifügen und angeben, ob Änderungen vorgenommen wurden.

\section{Literatur}

1. World Health Organisation (2011) WHO report on the global tobacco epidemic. http://www.who.int/ tobacco/global_report/2011/en/

2. Steppuhn H, Kuhnert R, Scheidt-Nave C (2017) 12 Monats-Prävalenz der bekannten chronisch obstruktiven Lungenerkrankung (COPD) in Deutschland. J Health Monit 2(3):46-54

3. Mons U, KahnertS (2017) Recalculation of tobaccoattributable mortality: national and regional data for Germany. Gesundheitswesen. https://doi.org/ $10.1055 / \mathrm{s}-0042-123852$

4. Jha P, Peto R (2014) Global effects of smoking, of quitting, and of taxing tobacco. N Engl J Med 370:60-68

5. Orth B (2016) Die Drogenaffinität Jugendlicher in der Bundesrepublik Deutschland 2015. Rauchen, Alkoholkonsum und Konsum illegaler Drogen: aktuelle Verbreitung und Trends. In: BZg A (Hrsg) BZgA-Forschungsbericht. BZgA, Köln

6. Doll R, Peto R, Wheatley K, Gray R, Sutherland I (1994) Mortality in relation to smoking: 40 years' observations on male British doctors. BMJ 309:901-911

7. Joossens L, Raw M(2017) The tobacco control scale 2016 in Europe. http://www.tobaccocontrolscale. org/wp-content/uploads/2017/03/TCS-2016-inEurope-COMPLETE-LoRes.pdf

8. Schaller K, Mons U (2016) Tabakwerbung auf Plakaten spricht Jugendliche an - Außenwerbeverbot dringend notwendig. https://www.dkfz.de/de/ tabakkontrolle/download/Publikationen/AdWfP/ AdWfdP_2016_Tabakwerbung-auf-Plakaten_ final.pdf

9. Deutsches Krebsforschungszentrum (2015) Tabakatlas Deutschland 2015

10. Brinker TJ, Stamm-Balderjahn S, Seeger W, Klingelhofer D, Groneberg DA (2015) Education Against Tobacco (EAT): a quasi-experimental prospective evaluation of a multinational medical-studentdelivered smoking prevention programme for secondary schools in Germany. BMJ Open 5:e8093

11. Brinker TJ, Seeger W, Buslaff F (2016) Photoaging mobile apps in school-based tobacco prevention: the mirroring approach.J Med Internet Res 18:e183

12. Brinker TJ, Seeger W (2015) Photoaging mobile apps: a novel opportunity for smoking cessation? JMed Internet Res 17:e186

13. Xavier LE, Bernardes-Souza B, Lisboa OC etal (2017) A medical student-delivered smoking prevention program, educationagainst tobacco, for secondary schools in Brazil: study protocol for a randomized trial.Jmir Res Protoc 6:e16

14. Haustein KO, Groneberg D (2010) Tobacco or health? Physiological and social damages caused by tobacco smoking. Springer, Berlin Heidelberg

15. World Health Organisation (2016) Growing up unequal: gender and socioeconomic differences in yound people's health and well-being. Health Policy Child Adolesc 7:151

16. Kuntz B, Lampert T (2016) Smoking and passive smoke exposure among adolescents in Germany- prevalence, trends over time, and differences between social groups. Dtsch Arztebl Int 113(3):23-30

17. Kraus L, Baumeister S, Stonner T (2008) 2006 epidemiological survey on substance abuse in the adult population of Bavaria. IFT-Reports, Bd. 162

18. Scholz M, Kaltenbach M (2000) Promoting nonsmoking behavior in 13-year-old students in primary schools and high schools. A prospective, randomized intervention study with 1,956 students. Gesundheitswesen 62:78-85

19. Stamm-Balderjahn S, Groneberg DA, Kusma B, Jagota A, Schonfeld N (2012) Smoking prevention in school students: positive effects of a hospitalbased intervention. Dtsch Arztebl Int 109:746-752

20. Sack PM, Hampel J, Bröning S et al (2013) Was limitiert schulische Tabakprävention? Ergebnisse aus "Nichtrauchen ist cool!" für 5. und 6. Klassen. Präv Gesundheitsf 4. https://doi.org/10.1007/ s11553-013-0388-z

21. Thomas RE, Mclellan J, Perera R (2013) Schoolbased programmes for preventing smoking. Cochrane Database Syst Rev. https://doi.org/10. 1002/14651858.CD001293.pub3:Cd001293

22. Muller BC, Ritter SM, GlockS, Dijksterhuis A, Engels RC, Van Baaren RB (2016) Smoking-related warning messages formulated as questions positively influence short-term smoking behaviour. J Health Psychol 21:60-68

23. Baudson TG, Weber KE, Freund PA (2016) More than only skin deep: appearance self-concept predicts most of secondary school students' self-esteem. Front Psychol 7:1568

24. Burford O, Jiwa M, Carter O, Parsons R, Hendrie D (2013) Internet-based photoaging within Australian pharmacies to promote smoking cessation: randomized controlled trial. J Med Internet Res 15:e64

25. Okada HC, Alleyne B, Varghai K, Kinder K, Guyuron B (2013) Facial changes caused by smoking: a comparison between smoking and nonsmoking identical twins. Plast Reconstr Surg 132:1085-1092

26. Brinker TJ, Enk A, Gatzka M et al (2017) A dermatologist's ammunition in the war against smoking: a photoaging app. J Med Internet Res 19:e326

27. Kempf D, Koldampf-Wendel A (2014) Kinder und Jugend 3.0. https://www.bitkom.org/ Presse/Anhaenge-an-PIs/2014/April/BITKOMPK-Kinder-und-Jugend-3-0.pdf

28. McEachan RRC, Conner M, Taylor NJ, Lawton RJ (2011) Prospective prediction of health-related behaviours with the theory of planned behaviour: a meta-analysis. Health Psychol Rev 5:97-144

29. Mons U, Müezzinler A, Gellert C et al (2015) Impact of smoking and smoking cessation on cardiovascular events and mortality among older adults: meta-analysis of individual participant data from prospective cohort studies of the CHANCES consortium. BrMed J350. https://doi.org/10.1136/ bmj.h1551

30. Bernstein SL, Yu S, Post LA, Dziura J, Rigotti NA (2013) Undertreatment of tobacco use relative to other chronic conditions. Am J Public Health 103:e59-e65

31. Raupach T, Falk J, Vangeli E et al (2014) Structured smoking cessation training forhealth professionals on cardiology wards: a prospective study. Eur J Prev Cardiol 21:915-922 
32. Anders S, Strobel L, Krampe H, Raupach T (2013) Do final-year medical students know enough about the treatment of alcohol use disorders and smoking? Dtsch Med Wochenschr 138:23-27

33. Twardella D, Brenner H (2005) Lack of training as a central barrier to the promotion of smoking cessation: a survey among general practitioners in Germany. Eur JPublic Health 15:140-145

34. Vitoria PD, Silva SA, Vries HD (2011) Longitudinal evaluation of a smoking prevention program for adolescents. Rev Saude Publica 45:344-354

35. Raupach T, Strobel L, Beard E, Krampe H, Anders S, West R (2013) German medical students' beliefs about the effectiveness of different methods of stopping smoking. Nicotine Tob Res 15:1892-1901

36. Strobel L, Schneider NK, Krampe H et al (2012) German medical students lack knowledge of how to treat smoking and problem drinking. Addiction 107:1878-1882

37. Brinker TJ, Stamm-Balderjahn S, Seeger W, Groneberg DA (2014) Education Against Tobacco (EAT): a quasi-experimental prospective evaluation of a programme for preventing smoking in secondary schools delivered by medical students: a study protocol. BMJ Open 4:e4909

38. Roseby R, Marks MK, Conn J, Sawyer SM (2003) Improving medical student performance in adolescent anti-smoking health promotion. Med Educ 37:704-708

39. Mays D, Niaura RS, Evans WD, Hammond D, Luta G Tercyak KP (2015) Cigarette packaging and health warnings: the impact of plain packaging and message framing on young smokers. Tob Control 24:e87-e92

40. Kreuter M, Bauer CM, Ehmann M, Kappes J, Drings $P$, Herth FJ (2014) Efficacy and sustainability of a smoking prevention programforpupils- „ohnekippe". Dtsch Med Wochenschr 139:1403-1408

41. Thrul J, Bühler A, Herth FJF (2014) Prevention of teenage smoking through negative information giving, a cluster randomized controlled trial. Drugs: EducPrev Policy 21:35-42

42. Kok G, Bartholomew LK, Parcel GS, Gottlieb $\mathrm{NH}$, Fernandez ME (2014) Finding theory- and evidence-based alternatives to fear appeals: intervention mapping. Int J Psychol 49:98-107

43. Brinker TJ, Owczarek AD, Seeger W et al (2017) A medical student-delivered smoking prevention program, education against tobacco, for secondary schools in Germany: randomized controlled trial. JMed Internet Res 19:e199

44. Johnston V, Liberato S, Thomas D (2012) Incentives for preventing smoking in children and adolescents. Cochrane Database Syst Rev 10:Cd8645

45. Brinker TJ, Holzapfel J, Baudson TG et al (2016) Photoaging smartphone app promoting poster campaign to reduce smoking prevalence in secondary schools: the Smokerface Randomized Trial: design and baseline characteristics. BMJ Open 6:e14288

46. DeVriesH,DijkF, Wetzels Jetal (2006)TheEuropean Smoking prevention Framework Approach (ESFA): effects after 24 and 30 months. Health Educ Res 21:116-132

47. Faria BL, Brieske CM, Cosgarea I et al (2017) A smoking prevention photoageing intervention for secondary schools in Brazil delivered by medical students: protocol for a randomised trial. BMJ Open 7:e18589

48. Brinker T, Alfitian J, Seeger W, Groneberg D, von Kalle C, Enk A, Suhre J et al (2018) A faceaging smoking prevention/cessation intervention for nursery school students in Germany: An appearance-focused interventional study. Int JEnv Res PubHe 15(8):1656 